\title{
A Novel Approach for Designing Omnidirectional Slotted-Waveguide Antenna Arrays
}

\author{
H.R.D. Filgueiras ${ }^{1}$, I. F. da Costa $^{1}$, Arismar Cerqueira S. Jr. ${ }^{1}$, James R. Kelly ${ }^{2}$ and Pei Xiao ${ }^{2}$ \\ ${ }^{1}$ Laboratory WOCA, National Institute of Telecommunication (Inatel), Santa Rita do Sapucaí, Brazil. \\ ${ }^{2} \mathrm{ICS} / 5 \mathrm{GIC}$, The University of Surrey, Stag Hill Campus, Guildford, GU2 7XH, UK. \\ hugorodrigues@get.inatel.br, arismar@inatel.br
}

\begin{abstract}
This paper presents a novel design of a high-gain omnidirectional slotted-waveguide antenna array for $5 \mathrm{G} \mathrm{mm-}$ wave applications. The structure is based on a circular waveguide filled with teflon for manipulating its dimension. It provides 12,1 dBi gain and omnidirectional coverage in the azimuth plane with only $1.3 \mathrm{~dB}$ deviation, which is ensured by making use of a twisting technique for proper placing the slots into the waveguide walls. A bandwidth of $1.61 \mathrm{GHz}$ centered at 26.2 GHz has been numerically demonstrated.
\end{abstract}

Index Terms - 5G networks, antenna array, mm-waves, slotted-waveguide antenna arrays.

\section{INTRODUCTION}

New applications, including video streaming, real time communications and online games cause a significant data demand in the current wireless network. The fifth-generation of cellular networks $(5 \mathrm{G})$ is envisioned to meet this demand, by supporting broadband transmission with bit rate up to 50 Gbits/s [1]. Larger bandwidths are required to provide those bit rates and overcome the spectrum scarcity problem. In this context, the application of mm-wave communications has been shown as a potential promising solution, since this frequency range is still unexplored [2].

Slotted-waveguide antenna arrays (SWAA) have been considered as a potential solution for mm-wave applications. W.H. Watson et al. were one of the first to present slots as resonant antennas in the literature in 1946. It has been demonstrated that if the current flow is properly disturbed into the waveguide wall, the slots will be excited and a radiation to space takes place [3]. This wave disturbance inside the waveguide makes the slots act as a dipole and, consequently, the entire structure functions as a dipole antenna array. Posterior, R. S. Elliot presented an equivalent circuit of a rectangular slotted-waveguide antenna array [4]. The main advantages of this design

Our research group has been developing SWAAs for diverse applications. In [5], a dual-polarization and dual-band SWAA operating at 2.5 and $4.9 \mathrm{GHz}$ was proposed for dualuse radar applications. In [6], a dual-band rectangular waveguide-based antenna array was idealized for $5 \mathrm{G}$ indoor networks operating in the 28 and $38 \mathrm{GHz}$ bands. Two SWAAs for $5 \mathrm{G} \mathrm{mm-wave} \mathrm{applications,} \mathrm{a} \mathrm{dual-band} \mathrm{rectangular}$ waveguide-based and a ring-shaped leaky-wave antenna, were presented by our group in [7]. At the beginning of this year, we reported the first optically controlled antenna for mmwaves [8]. We also presented a ring-shaped mechanically reconfigurable SWAA for 5G networks [9], which was based on the omnidirectional leaky-wave antenna described in [10].

This paper introduces a novel approach for designing high-gain omnidirectional SWAA for 5G networks. We propose to employ it for radio access applications, as illustrated in Fig. 1. An omnidirectional coverage in an Olympic arena can be ensured by using only one high-gain radiating element. This is especially useful for multicast/broadcast transmissions.

The remainder of the manuscript is structured as follows. Section II presents the first version of the omnidirectional SWAA, as well as its simulations results. The novel approach based on a twisting technique, idealized for providing better omnidirectional behavior, is described in Section III. Finally, conclusions are drawn in Section IV.

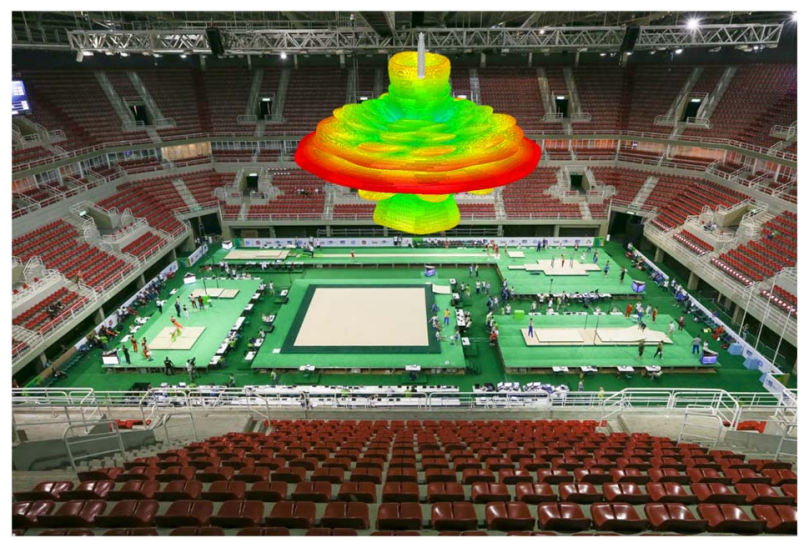

Fig.1. Omnidirectional SWAA for indoor mm-waves applications.

\section{SLOtTED-WAVEGUIDE ANTENNA ARRAY}

Slotted-waveguide antenna arrays (SWAAs) consist of a waveguide with slots milled into their walls in order to interrupt the current flow [3]. This wave disturbance inside the waveguide makes the slots to act as a dipole and, consequently, the entire structure functions as a dipole antenna array. Their main advantages are low loss, high polarization purity, high-power handling capability, simple design and fabrication as well as high gain when compared with a conventional printed antennas for high frequencies applications.

Fig. 2 shows the numerical model of the original SWAA version. A circular waveguide excited with a transverse 
magnetic (TM) mode was used for the SWAA design in order to guarantee an omnidirectional radiation pattern. The slots are placed orthogonally to the electric field inside de waveguide in order to properly interrupt the current flow in its wall and allow the radiation as a resonant antenna. Eight slots are placed around $\mathrm{z}$-axis for a $360^{\circ}$ beamwidth in the azimuth plane and then properly replicated through the $\mathrm{z}$-axis to increase the gain. The waveguide was filled with teflon, with the purpose of proper manipulating the impedance matching and reducing the slot size in order to fit it in the waveguide circumference.

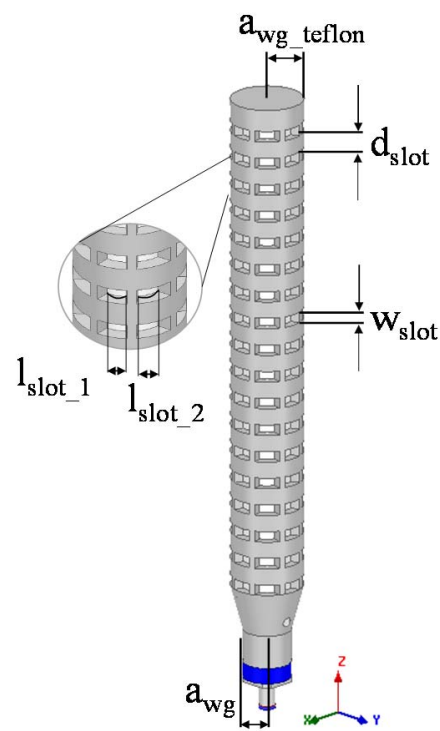

Fig. 2. The original numerical model of the SWAA.

A commercial circular waveguide (WC 38) with $\mathrm{TM}_{01}$ mode cut off frequency of $24.11 \mathrm{GHz}$ and radius $\mathrm{a}_{\mathrm{wg}}=4.7625$ $\mathrm{mm}$ was designed for exciting the proposed SWAA. The radius of the SWAA top part ( $\left.\mathrm{a}_{\mathrm{wg} \_ \text {teflon }}\right)$, i.e. the one filled with teflon, was increased to $8.74 \mathrm{~mm}$, another commercial waveguide (WC 69), for enabling us to add eight slots of approximately lambda over two. Two distinct slots length were designed in order to increase the array bandwidth by creating two different resonant frequencies. The slots length ( $1_{\text {slot_1 }}$ e $\left.1_{\text {slot_2}}\right)$, width ( $\left.\mathrm{w}_{\text {slot }}\right)$ and spacing $\left(\mathrm{d}_{\text {slot }}\right)$ were optimized using ANSYS HFSS. The SWAA final dimensions were: $1_{\text {slot_ } 1}=4.2 \mathrm{~mm} ; 1_{\text {slot_ } 2}=4.7 \mathrm{~mm} ; \mathrm{w}_{\text {slot }}=3.5 \mathrm{~mm} ; d_{\text {slot }}=5.4 \mathrm{~mm}$.

Fig. 3 presents the SWAA first version reflection coefficient $\left(\mathrm{S}_{11}\right)$, in which one can observe a $1.46 \mathrm{GHz}$ bandwidth centered at $26.2 \mathrm{GHz}$, which corresponds to a fractional bandwidth of $5.57 \%$ for S1 lower than $-10 \mathrm{~dB}$. Fig. 4 displays the normalized radiation pattern in the azimuth plane (xy) and elevation plane (yz) at $26 \mathrm{GHz}$. The SWAA provides a $5^{\circ}$ beamwidth and $8.55 \mathrm{~dB}$ side lobe level (SLL) in the elevation plane. On the other hand, in the azimuth plane, we have four lobes with $22^{\circ}$ of beamwidth equally distributed around the antenna structure and for secondary lobes with $\mathrm{SLL}=4.2 \mathrm{~dB}$ placed between each main lobe. The SWAA achieved gain is $16 \mathrm{dBi}$ at $26 \mathrm{GHz}$.

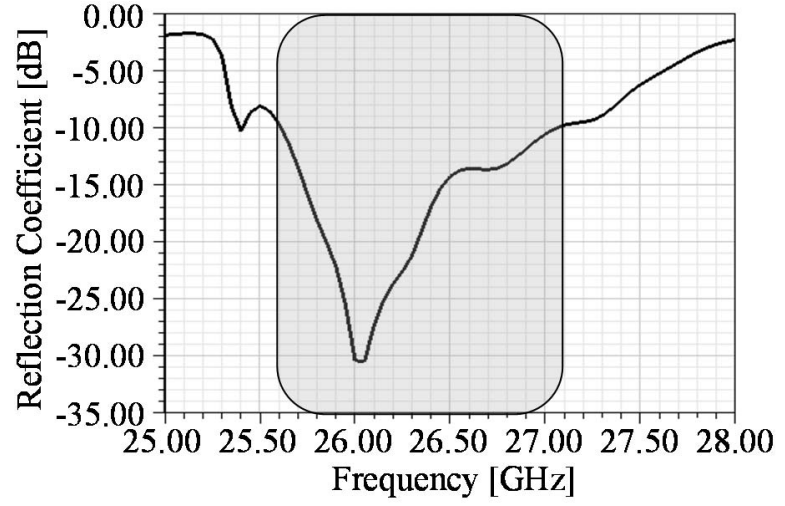

Fig. 3. Reflection Coefficient of the SWAA.

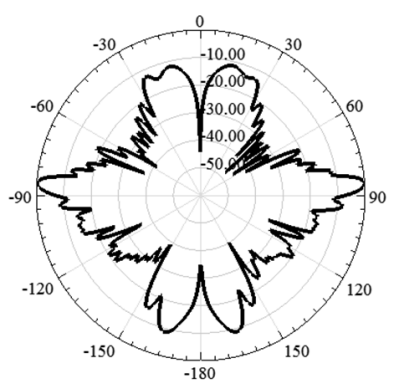

(a) Elevation plane $(y z)$

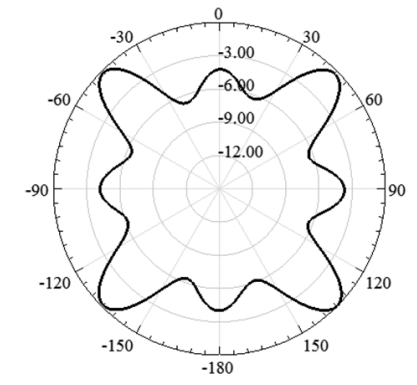

(b) Azimuth plane (xy)
Fig. 4. SWAA normalized radiation patterns at $26 \mathrm{GHz}$.

\section{THE NOVEl OMNIDIRECTIONAL SLOTTED- WAVEGUIDE ANTENNA ARRAY DESIGN}

To improve the performance, we apply a twisting technique in the slotted-waveguide structure, as illustrated in Fig. 5, with the purpose of refining the omnidirectional behavior, by means of reducing the gain deviation in the array azimuth plane. We define the angle difference between two consecutive levels of slots in the z-axis as $\alpha$; it is possible to return the SWAA original version presented in Section II by setting $\alpha=0^{\circ}$. The SWAA novel design numerical model is depicted in Fig. 6.

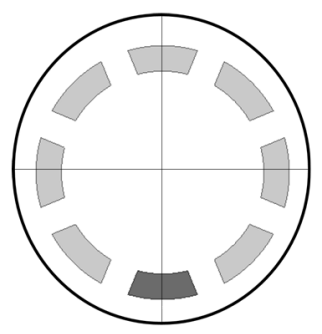

(a) Level (i) of slots.

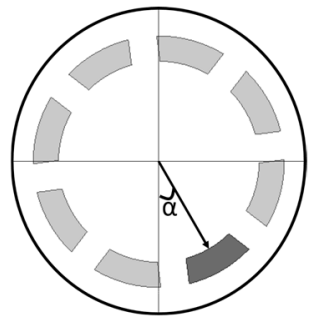

(b) Level $(i+1)$ of slots rotated of $\alpha$ degrees.
Fig. 5. Twisting technique definition. 


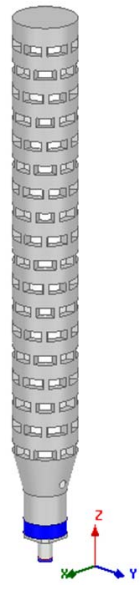

Fig. 6. Novel Omnidirectional SWAA numerical model.

The numerical results of the SWAA reflection coefficient for different values of $\alpha$ are reported in Fig. 7, which demonstrates this parameter does not significantly change the antenna impedance matching. Although, we can observe a slightly bandwidth increase from 1.46 to $1.61 \mathrm{GHz}$ for $\alpha=$ $22.5^{\circ}$, which correspond to $6.15 \%$ for S11 lower than $-10 \mathrm{~dB}$. It is observed a variation of only $180 \mathrm{MHz}$ over the $1.61 \mathrm{GHz}$ bandwidth centered at around $26.2 \mathrm{GHz}$ for different values of $\alpha$. Fig. 8 presents the normalized radiation pattern at $26 \mathrm{GHz}$ in the elevation and azimuth planes for the following values of $\alpha: 0^{\circ}, 10^{\circ}$ and $22.5^{\circ}$, which yields the best achieved result. In the elevation plane, the $5^{\circ}$ half-power beamwidth has been kept for all three cases, while the SLL is $-8.55 \mathrm{~dB}$ for $\alpha=0^{\circ}$, $-8 \mathrm{~dB}$ for $10^{\circ}$ and $-10.65 \mathrm{~dB}$ for $22.5^{\circ}$. On the other hand, the radiation pattern tends to become omnidirectional as $\alpha$ increases from $0^{\circ}$ to $22.5^{\circ}$, since the deviation is gradually reduced, attaining $6.42,2.15$ and $1.3 \mathrm{~dB}$ for $0^{\circ}, 10^{\circ}$ and $22.5^{\circ}$, respectively. As expected, as the energy is uniformly distributed, the gain is slightly reduced from $16 \mathrm{dBi}$ for $\alpha=0^{\circ}$ to 12.6 and $12.1 \mathrm{dBi}$ for $10^{\circ}$ and $22.5^{\circ}$ respectively. In any case, the novel SWAA gain is almost ten times higher than that of a conventional half-wave dipole antenna, that is only $2.15 \mathrm{dBi}$.

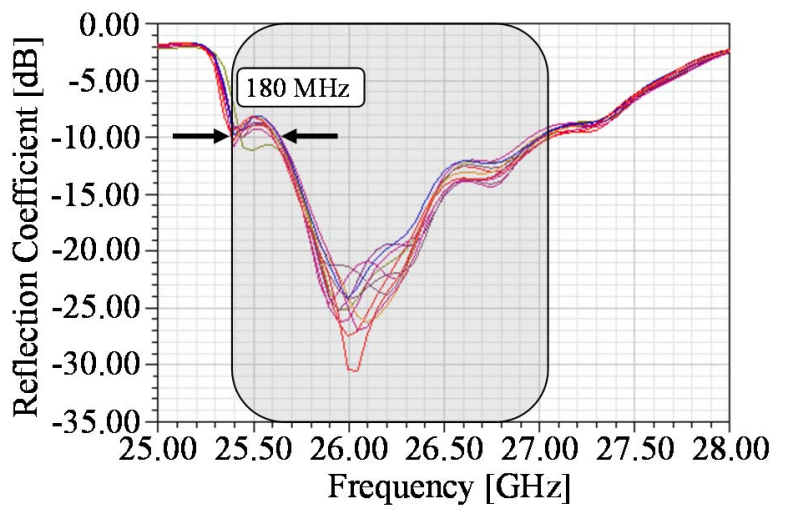

Fig. 7. Reflection coefficient as a function of $\alpha$ (from $0^{\circ}$ to $20^{\circ}$ ).

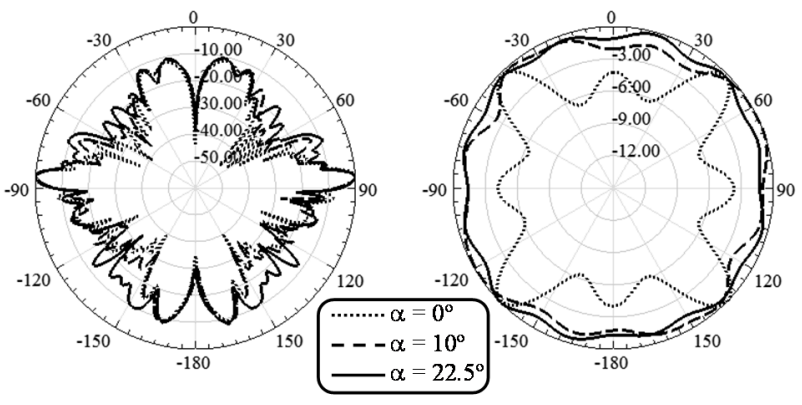

(a) Elevation plane

(b) Azimuth plane

Fig. 8. Radiation pattern at $26 \mathrm{GHz}$ as a function of $\alpha$

We have manufactured a prototype with milled aluminum and envisage its characterization and implementation in real systems operating with broadcast communication at $\mathrm{mm}$ wave as future works. Fig. 9 is a photograph of the constructed prototype.

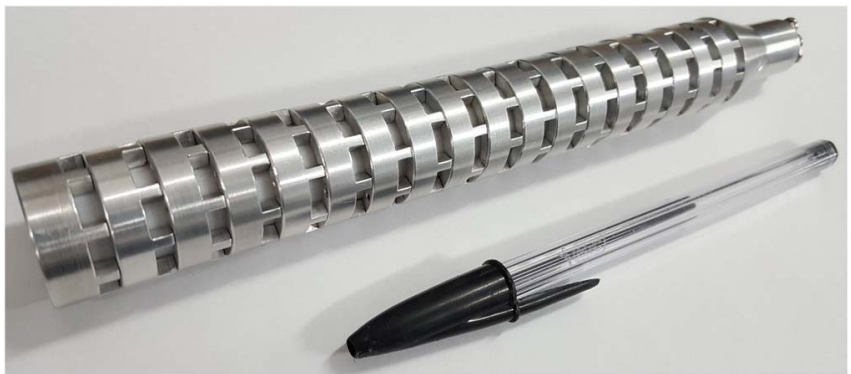

Fig. 9. Omnidirectional SWAA prototype.

\section{CONCLUSIONS}

We have proposed a novel approach for designing highgain omnidirectional slotted-waveguide antenna arrays for $5 \mathrm{G}$ mm-wave applications. Our technique consists of proper milling a circular waveguide with slots strategically placed into its walls in order to guarantee an omnidirectional radiation pattern. The novelty of the design comes from the use of a twisting technique between consecutive levels of slots. The proposed array provides the following properties: a bandwidth of $1.61 \mathrm{GHz}$ centered at $26.2 \mathrm{GHz}(6.15 \%)$; gain of $12.1 \mathrm{dBi}$ at $26 \mathrm{GHz}$; beamwidth of $5^{\circ}$ in the elevation plane; omnidirectional radiation pattern in the azimuth plane with only $1.3 \mathrm{~dB}$ variation. This technique is considered a potential solution for communication systems at malls, stadiums, arenas and universities to name a few, which makes it a promising radiating structure for $5 \mathrm{G}$ small cell networks. As future works, we envisage the SWAA prototype characterization to validate the numerical simulation results and implement it in a real $5 \mathrm{G}$ network operating in $\mathrm{mm}$-wave bands.

\section{ACKNOWLEDGMENTS}

This work was partially supported by Finep/Funttel Grant No. 01.14.0231.00, under the Radio Communications Reference Centre (CRR) project of the National Institute of Telecommunications (Inatel), Brazil. Authors also thank the financial support from CNPq, CAPES, MCTI and FAPEMIG 
and technical support from Keysight and ESSS-ANSYS.

Authors from the UK would like to acknowledge the support of the University of Surrey 5GIC (http://www.surrey.ac.uk/5gic) members for this work.

\section{REFERENCES}

[1] DMC R\&D Center, Samsung Electronics Co., Ltd., "5G Vision” (white paper), February, 2015.

[2] T. S. Rappaport, S. Sun, R. Mayzus, H. Zhao, Y. Azar, K. Wang, G. N. Wong, J. K. Schulz, M. Samini and F. Gutierrez, "Millimeter Wave Mobile Communication for 5G Cellular: It Will Work!", IEEE Access, vol. 1, pp. 335-349, May 29, 2013.

[3] W. H. Watson, "Resonant Slots", Journal of the Institution of Electrical Engineers, vol. 93, pp. 747-777, April 10, 1946.

[4] J. J. Gulick, G. J. Stern and R. S. Elliott, "The Equivalent Circuit of a Rectangular-Waveguide-Fed Longitudinal Slot”, Antennas and Propagation Society International Symposium, Philadelfia, USA, 1986.

[5] Arismar Cerqueira S. Jr., I.F. da Costa, Pinna, S., S. A. de S.Melo, Laghezza, F., Scotti, F., Ghelfi, P., Spadoti, D. H., A. Bogoni. "A Novel Dual-polarization and Dual-band Slotted Waveguide Antenna Array for Dual-use Radars." in 10th European Conference on Antennas and Propagation (EUCAP), Davos, Switzerland, 2016.

[6] I. F. da Costa, Arismar Cerqueira S. Jr., D. H. Spadoti, "Dual-band slotted waveguide antenna array for adaptive mm-wave 5G networks", in 11th European Conference on Antennas and Propagation (EUCAP), Paris, France, 2017.

[7] Arismar Cerqueira Sodré Jr., Igor Feliciano da Costa, Renan Alves dos Santos, Hugo Rodrigues Dias Filgueiras, and Danilo Henrique Spadoti, "Waveguide-Based Antenna Arrays for 5G Networks," International Journal of Antennas and Propagation, vol. 2018, Article ID 5472045, 10 pages, 2018. doi:10.1155/2018/5472045

[8] I. F. da Costa, Arismar Cerqueira S. Jr, D. H. Spadoti, L. G. da Silva, J. A. J. Ribeiro, S. E. Barbin, "Optically Controlled Reconfigurable Antenna Array for mm-wave Applications", IEEE Antennas and Wireless Propagation Letters (AWPL), vol. 16, pp. 2142-2145, May 02, 2017.

[9] H.R.D. Filgueiras, I. F. da Costa, R. A. Santos, James R. Kelly, Arismar Cerqueira S. Jr., "Mechanically Reconfigurable Slotted-Waveguide Antenna Array for 5G Networks", International Microwave and Optoelectronics Conference (IMOC), 2017.

[10] Shanjia Xu, Jianhua Min, Song-Tsuen Peng and F. K. Schwering, "A millimetre-wave omnidirectional circular dielectric rod grating antenna," in IEEE Transactions on Antennas and Propagation, vol. 39, no. 7, pp. 883-891, July, 1991 